Einführung zum Thema

Knie J. $2020 \cdot 2: 65-66$

https://doi.org/10.1007/s43205-020-00060-1

(C) Springer Medizin Verlag GmbH, ein Teil von Springer Nature 2020

\section{J. Raschke' $\cdot$ S. Schröter ${ }^{2} \cdot$ E. Herbst $^{1}$}

'Klinik für Unfall-, Hand- und Wiederherstellungschirurgie, Universitätsklinikum Münster, Münster, Deutschland

${ }^{2}$ Unfall- und Wiederherstellungschirurgie, Diakonie Klinikum GmbH, Jung-Stilling-Krankenhaus, Siegen, Deutschland

\title{
Neue Konzepte am Tibiaplateau - oder Altbekanntes?
}

Die Neugier steht immer an erster Stelle eines Problems, das gelöst werden will. (Galileo Galilei, 1564-1642)

Gemäß diesem Zitat von Galileo Galilei sind in den vergangenen Jahren Tibiaplateaufrakturen zunehmend in den Fokus wissenschaftlicher Arbeiten gerückt. Dies zeigt sich, wenn man beispielsweise in PubMed (https://www.ncbi.nlm.nih. gov/pubmed) nach „tibial plateau fracture" sucht. So gibt es unter diesem Suchbegriff 18 Einträge für das Jahr 2012, 35 für 2014 und in den letzten drei Jahren stets über 60. Im Vergleich zu anderen Bereichen am Kniegelenk ist die Zahl an Neupublikationen relativ gering, lässt aber deutlich das Interesse am Tibiaplateau erkennen. Die Frage, nach dem Problem, das gelöst werden will, kann man am Tibiaplateau in viele Richtungen beantworten.

So wurde in den letzten Jahren zunehmend Altbekanntes überarbeitet bzw. in einen neuen Kontext gebracht. Die geläufigen Klassifikationssysteme nach Schatzker oder Moore, welche auf zweidimensionaler Röntgendarstellung basieren [6, 8], werden durch dreidimensionale Klassifikationen ergänzt [3,5]. Der Vorteil dieser Klassifikationen liegt nicht nur im besseren Verständnis für die Frakturmorphologie, sondern insbesondere in der präoperativen Planung der Zugangswege und Wahl der Osteosyntheseverfahren $[4,7]$.

Bei der Diagnostik von Tibiaplateaufrakturen gilt die Computertomographie (CT) aus vorgenannten Gründen als Standard. Nach wie vor wird darüber diskutiert, ob und wann eine Gefäßdar- stellung notwendig ist. Neben klinischen Kriterien (Pulsstatus, Knöchel-Arm-Index, Doppler-Sonographie) empfehlen wir die großzügige Indikationsstellung zur CT-Angiographie, da eine Vielzahl komplexer Tibiaplateaufrakturen im Rahmen von Hochrasanztraumta entstehen bzw. Luxationsfrakturen sind [1]. Im Zuge dessen rückt auch die Magnetresonanztomographie (MRT) zunehmend in den Vordergrund, um therapiebedürftige Begleitpathologien an Bändern und Menisken präoperativ zu detektieren. Allerdings ist die MRT zeit- und kostenaufwendig, was die Verfügbarkeit deutlich reduziert. Daher empfehlen wir intraoperativ die dynamische Durchleuchtung, um relevante Instabilitäten der Kreuz- und Kollateralbänder auszuschließen.

In diesem Kontext gewinnt auch die arthroskopisch gestützte Osteosynthese zunehmend an Bedeutung. Einerseits können hierdurch Meniskuspathologien besser detektiert und behandelt werden, andererseits können schwer einsehbare Bereiche des Tibiaplateaus unter Sicht reponiert werden. Hierdurch kann sich künftig die Versorgungsqualität und dadurch potenziell das Langzeitergebnis verbessern. Dies gilt insbesondere vor dem Hintergrund, dass die aktuelle Evidenz Gelenkstufen von bis $\mathrm{zu} 10 \mathrm{~mm}$ am Tibiaplateau erlaubt [2]. Allerdings beruhen diese Daten auf teilweise historischen Arbeiten mit relevanten methodischen Mängeln. Hier besteht sicherlich noch Nachholbedarf, sowohl was biomechanische als auch klinische Daten anbelangt.
Zusammenfassend kann man sagen, dass es nach wie vor große wissenschaftliche Lücken am Tibiaplateau gibt, welche sich teils auch in der Versorgungsqualität widerspiegeln. Allerdings haben zahlreiche Arbeitsgruppen in den letzten Jahren einen wesentlichen Beitrag geleistet, um das Verständnis zu steigern und dadurch die Operationsstrategien zu optimieren. Die folgende Ausgabe des Kniejournals wird Ihnen alles Relevante zum Tibiaplateau in kompakter Form darstellen, damit auch Sie einen Beitrag dazu leisten, aus der Neugier heraus Probleme zu lösen.

Mit freundlichen Grüßen,

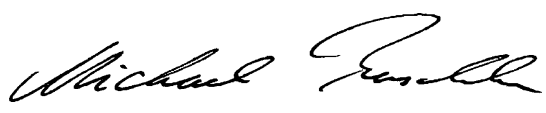

Univ.-Prof. Dr. med. Michael J. Raschke

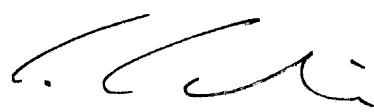

Prof. Dr. med. Steffen Schröter

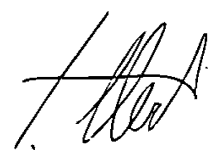

Dr. med. univ. Elmar Herbst, PhD 


Univ.-Prof. Dr. med.
M. J. Raschke
Klinik für Unfall-, Hand- und
Wiederherstellungschirurgie,
Universitätsklinikum Münster
Albert-Schweitzer-Campus 1,
48147 Münster, Deutschland
michael.raschke@
ukmuenster.de

Interessenkonflikt. M.J. Raschke, S. Schröter und E. Herbst geben an, dass kein Interessenkonflikt besteht.

\section{Literatur}

1. Elsoe R, Larsen P, Nielsen NP, Swenne J, Rasmussen S, Ostgaard SE (2015) Population-based epidemiology of tibial plateau fractures. Orthopedics 38:e780-e786

2. Giannoudis PV, Tzioupis C, Papathanassopoulos A, Obakponovwe O, Roberts C (2010) Articular step-off and risk of post-traumatic osteoarthritis. Evidence today. Injury 41:986-995

3. Kfuri M, Schatzker J (2018) Revisiting the Schatzker classification of tibial plateau fractures. Injury 49:2252-2263

4. Krause M, Kruger S, Muller G, Puschel K, Frosch KH (2019) How can the articular surface of the tibial plateau be best exposed? A comparison of specific surgical approaches. Arch Orthop Trauma Surg 139:1369-1377

5. Krause M, Preiss A, Muller G, Madert J, Fehske K, Neumann MV et al (2016) Intra-articular tibial plateau fracture characteristics according to the "Ten segment classification". Injury 47:2551-2557

6. Moore TM (1981) Fracture-dislocation of the knee. Clin Orthop Relat Res (156):128-140

7. Raschke MJ, Kittl C, Domnick C (2017) Partial proximal tibia fractures. EFORT Open Rev 2:241-249

8. Schatzker J (1974) Compression in the surgical treatment of fractures of the tibia. Clin Orthop Relat Res 105:220-239

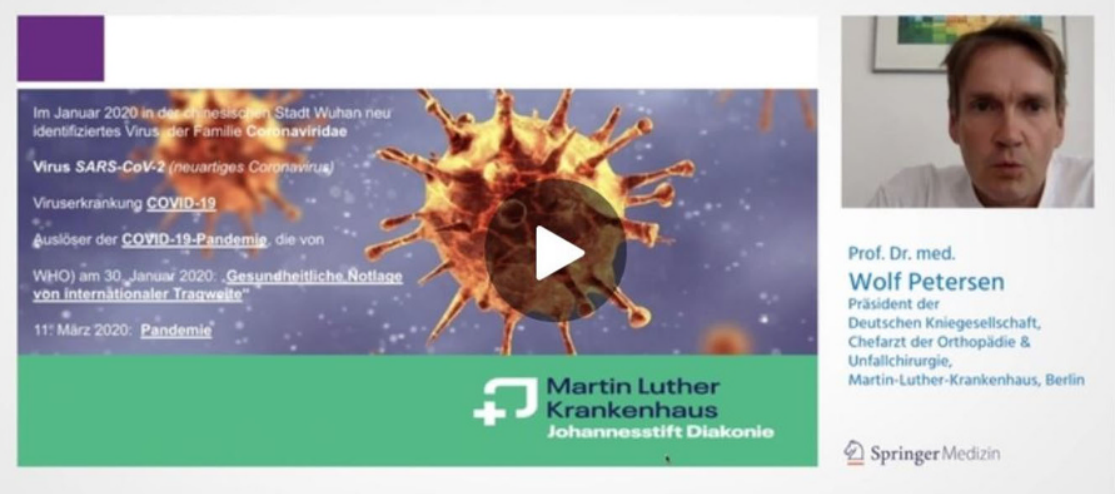

\section{Corona-Webinare bei Springer Medizin}

\section{Webinare und Videointerviews über das Ärzteportal SpringerMedizin.de}

Springer Medizin hat seit April die Initiative Corona-Webinar über sein Ärzteportal (www.springermedizin.de) gestartet. Ärzte, die auf diesem Portal registriert sind, gelangen über Videoseminare an kostenloses Wissen rund um das CoronaVirus. In jedem Webinar oder Videointerview kommt ein Experte zu einem bestimmten Thema zu Wort und gibt fachlich gesichertes Wissen an Kollegen weiter, die sich mit Symptomatik, Diagnostik und Therapie in Klinik und Praxis beschäftigen. Wöchentlich kommen neue Themen hinzu!

Auf der zentralen Corona-UpdateSeite auf SpringerMedizin.de sind unter anderem bereits folgende Webinare verfügbar:

- Orthopädisch-unfallchirurgische Operationen während der COVID-19Pandemie

- Notfallplanung: So begegnete "Hotspot" Rosenheim der Corona-Pandemie

- Notfallversorgung in Zeiten von COVID19

- SARS-CoV-2-Testverfahren: Wie Sie funktionieren, was sie leisten und was nicht

- Impfstoffe und Therapien gegen SARSCoV-2: Was kann welcher Kandidat?

- Infektiologe über SARS-CoV-2: "Wir werden dieses Virus nicht ewig bei uns haben"

- COVID-19-Pandemie: Aktuelles zu Epidemiologie, Diagnostik und Therapiestrategien

- Covid-19 in der Klinik: "Fast Progressors", klinische Charakteristika und prognostische Marker
Alle Webinare sind über www.springermedizin.de/covid-19 zugänglich, die Seite wird ständig aktualisiert und erweitert.

„In unserer Ärztekommunikation werden wir das Webinar-Format zukünftig stärker nutzen, denn es ist eine sehr wertvolle Ergänzung zu den bestehenden Print- und OnlineAngeboten", erläutert Cécile Mack, Director Digital Product \& Marketing von Springer Medizin. „Die Inhalte eines Webinars oder VideoInterviews können durch die direkte Ansprache schneller aufgenommen werden. Unsere Redakteure der verschiedenen Magazine und Zeitschriften laden zum Ausbau dieser Initiative ausgewählte Autoren und Herausgeber ein, ihr Fachwissen zu Corona zu teilen. Neben den aktuellen Aspekten werden wir auch in den nächsten Monaten das Thema eng begleiten und über die Erkenntnisse aus klinischen Studien sowie der Entwicklung von Therapieoptionen und Impfstoffen berichten. Solange die SARS-CoV-2/Covid-19-Pandemie eine Bedrohung unserer Gesellschaft darstellt, wird Springer Medizin als ein führender Marktvertreter diese Inhalte kostenfrei vor der Bezahlschranke platzieren, um allen Ärzten eine sichere und fundierte Wissensbasis zu geben. Denkbar ist außerdem, dieses Format auch auf andere medizinische Themen oder zusätzliche Fachgebiete auszudehnen", so die Digitaldirektorin. 